\section{Endoscopic ultrasound-guided ethanol lavage of a pancreatic fluid collection}

A 30-year-old man with a history of alcohol abuse developed a large pseudocyst $(89 \times 78 \mathrm{~mm})$ in the tail of the pancreas (Figure 1) following a single episode of acute severe pancreatitis with multiple organ dysfunction syndrome. As the patient declined any form of surgery, endoscopic decompression of the pseudocyst was carried out with real-time endoscopic ultrasound (EUS) guidance (Figure 2) after informed consent had been obtained. With the patient under general anesthesia, EUS was conducted with a Pentax FG-36UA ultrasound endoscope (Pentax Europe, Ltd., Hamburg, Germany) using a curved-array transducer with fluoroscopic guidance [1]. The pseudocyst was visualized as a bulge on the posterior gastric wall, and the shortest access route (about $10 \mathrm{~mm}$ ) in which there were no interposed vessels was clearly identified. A 19-Fr needle (Cook Medical Inc., Bloomington, Indiana, USA) was introduced through the endoscope's working channel to puncture the pseudocyst and inject $30 \mathrm{ml}$ iopromide (Ultravist) contrast. No communication with the pancreatic duct was visible.

Aspiration was started, and after an apparent reduction in the volume of the pseudocyst by about $30 \%, 30 \mathrm{ml}$ of absolute ethanol diluted $1: 1$ with saline was injected and maintained for about 10 minutes. Aspiration then continued until EUS imaging showed that the cyst was completely empty [2]. Computed tomography $24 \mathrm{~h}$ later demonstrated no complications and confirmed that the procedure had been successful. Culture of the aspiration fluid identified Pseudomonas aeruginosa and Citrobacter freundii complex. Cytological examination did not show any neoplastic cells. The patient was discharged on the seventh day with no symptoms and with normal laboratory tests.

There have been several recent reports in the literature on successful treatment of pseudocysts with ethanol lavage under EUS guidance [3-5]. It is possible that in

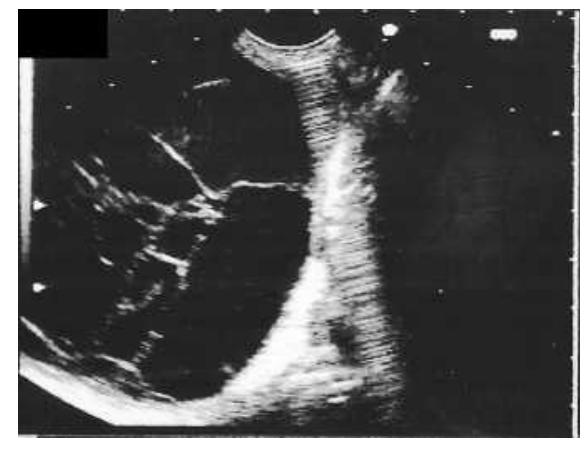

Figure 1 The pseudocyst is septate and contains debris. The aspiration needle is visible at the top of the image.

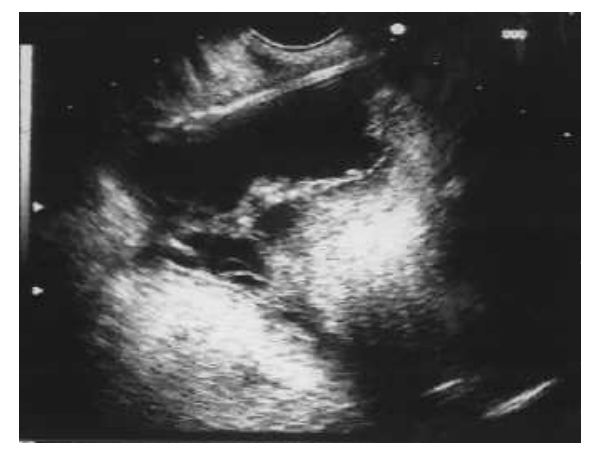

Figure 2 About one-third of the fluid collection has been aspirated before injection of the ethanol solution. Collapse of the cystic wall is visible.

addition to causing sclerosis of the cystic wall, ethanol contributes to sterilizing the infected fluid collection. In the present case, a long follow-up period (18 months) in which there was no recurrence of the pseudocyst confirms that this procedure may be useful in the treatment of organized necrotic abscesses and pancreatic abscesses when there is no communication with the pancreatic duct.

\section{Endoscopy_UCTN_Code_TTT_1AS_2AD}

N. Muscatiello', L. Pietrini', M. Gentile', P. Tonti' ${ }^{1}$ C. Ricciardelli', I. Sorrentini², E. Ierardi ${ }^{1}$

${ }^{1}$ Gastroenterology Unit, Azienda Ospedaliero Universitaria “Ospedali Riuniti,"

Foggia, Italy

2 Dept. of Gastroenterology and Interventional Endoscopy, Gaetano Rummo Hospital Benevento, Italy.

\section{References}

${ }^{1}$ Song MH, Lee SK, Kim MH et al. EUS in the evaluation of pancreatic cystic lesions. Gastrointest Endosc 2003; 57: 891 -896

${ }^{2}$ Gan SI, Thompson CC, Lauwers GY et al. Ethanol lavage of pancreatic cystic lesions: initial pilot study. Gastrointest Endosc 2005; 61: 746-752

${ }^{3}$ Vosoghi M, Sial S, Garrett B et al. EUS-guided pancreatic pseudocyst drainage: review and experience at Harbor-UCLA Medical Center. MedGenMed 2002; 4: 2

${ }^{4}$ Giovannini M, Pesenti C, Rolland AL et al. Endoscopic ultrasound-guided drainage of pancreatic pseudocysts or pancreatic abscesses using a therapeutic echo endoscope. Endoscopy 2001; 33: 473-477

${ }^{5}$ Siriam PV, Keffer AJ, Rao GV, Reddy DN. Endoscopic ultrasound-guided drainage of pancreatic pseudocysts complicated by portal hypertension or by intervening vessels. Endoscopy 2005; 37: 231 - 235

\section{Corresponding author}

\section{N. Muscatiello, M.D.}

Gastroenterology Unit

Azienda Ospedale Università

"Ospedali Riuniti"

Viale L. Pinto

71100 Foggia

Italy

Fax: $\quad+39-0881-733690$

E-mail: nmuscatiello@libero.it 